\title{
Assessments of vessel density and foveal avascular zone metrics in multiple sclerosis: an optical coherence tomography angiography study
}

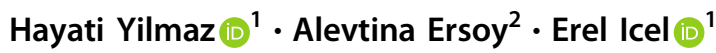

Received: 21 May 2019 / Revised: 9 October 2019 / Accepted: 11 November 2019 / Published online: 19 December 2019

(c) The Author(s), under exclusive licence to The Royal College of Ophthalmologists 2019

\begin{abstract}
Background/Objectives To investigate optical coherence tomography angiography (OCT-A) findings of foveal avascular zone (FAZ) metrics and macular \& peripapillary vessel densities (VD) in subjects with multiple sclerosis (MS).

Methods The study design was prospective and cross-sectional. FAZ metrics and VDs of the superficial capillary plexus (SCP), deep capillary plexus (DCP), retinal peripapillary capillary plexus (RPCP) along with the structural OCT measurements were scanned by using the Nidek's RS-3000 Advance in MS patients and healthy controls. All subject also underwent an assessment of visual evoked potentials (VEPs). The relationships between the OCT-A parameters with other clinical findings were analysed.

Results Forty-seven MS patients (94 eyes) and 61 healthy volunteers (122 eyes) were included in this study. Thirty-five eyes of the MS patients had an ON history. The structural OCT measurements were significantly differed between the groups $(P<0.001)$. All FAZ metrics were inversely correlated with central foveal thickness $(\mathrm{CFT})(P<0.001)$. The FAZ area and perimeter were inversely correlated with the VD of both SCP and DCP $(P<0.05)$. The VDs of SCP and DCP were significantly differed between the study groups $(P<0.001)$. The VEP latency was inversely correlated with the retinal nerve fibre layer, macular and ganglion cell layer thicknesses, the VD of SCP, and the VD of the DCP $(P<0.001)$.

Conclusions Based on OCT angiography, VDs of macular and peripapillary area may be useful in detecting damage from $\mathrm{ON}$ in patients with MS.
\end{abstract}

\section{Introduction}

Multiple sclerosis (MS) is both an autoimmune, inflammatory and a degenerative central nervous system disorder. Ocular involvement is frequent among MS patients [1-3]. Eighty percent of the patients have visual pathway involving including optic neuritis $(\mathrm{ON})$, which is an initial

Supplementary information The online version of this article (https:// doi.org/10.1038/s41433-019-0746-y) contains supplementary material, which is available to authorized users.

Hayati Yilmaz

dr.hyilmaz@hotmail.com

1 Department of Ophthalmology, Mengucek Gazi Training and Research Hospital, Binali Yildirim University, Erzincan, Turkey

2 Department of Neurology, Mengucek Gazi Training and Research Hospital, Binali Yildirim University, Erzincan, Turkey finding in 15-20\% of MS cases and occurs in $~ 50 \%$ of patients during the course of MS [1,2].

Visual evoked potentials (VEPs) and optical coherence tomography (OCT) are commonly used to investigate the visual pathway. A delayed latency and preserved morphology of VEPs are suggestive of the demyelinating disorder of the visual pathway. OCT provides information about the retinal nerve fibre layer (RNFL) and ganglion cell layer (GCL) and is discussed as a potential marker for monitoring disease severity of MS with or without $\mathrm{ON}$, and therapeutic response in individual patients [4-8].

In recent years, OCT angiography (OCT-A) is also used with increasing frequency to examine the visual pathway of patients with MS. Some studies suggested that retinal vascular density was reduced in this patient group [9-12]. However, to the best of our knowledge, no study has reported the foveal avascular zone (FAZ) metrics and their relations with vessel density (VD). Therefore, the aim of this study was to demonstrate the OCT-A characteristics, including the FAZ metrics of MS cases and investigate the 
relations with structural OCT, VEPs, expanded disability status scale (EDSS) score, and visual acuity.

\section{Materials and methods}

This prospective observational study was performed in the ophthalmology and neurology departments of Binali Yildirim University, Faculty of Medicine. The study protocol was approved by the institutional clinical research ethics committee and completed in agreement with the tenets of the Declaration of Helsinki. Written consent was obtained from all the participants after informing them about the nature of this study. From February 2019 to May 2019, 51 MS patients and 61 healthy volunteers were enroled in this study.

The inclusion criteria for all participants were being aged 18-70 years and having the best-corrected visual acuity (BCVA) of at least 20/200 in either eye. The MS diagnosis was confirmed by a neurologist in accordance of the 2010 McDonald Criteria [13], and only the patients with a relapsing-remitting subtype of MS (RR-MS) who had the EDSS score less than 6 (mobile without support) were included in this study. The exclusion criteria were presence of other ophthalmological pathologies that would confound the assessment results (glaucoma, diabetic or hypertensive retinal diseases, amblyopia, age-related macular degeneration, etc.), previous intraocular surgery, inability to cooperate OCT scanning, refractive error greater than \pm 6 dioptres, use of corticosteroids, or MS exacerbation in the past 30 days.

\section{Clinical data}

The duration of MS, medication used, relapse count, EDSS score, and self-recorded ON were obtained for all MS participants. The history of $\mathrm{ON}$ was confirmed with medical records. All participants underwent a full ophthalmological examination, including autorefraction (Tonoref III, Nidec Co. Ltd, Aichi, Japan), BCVA with a Snellen chart (converted to the $\log$ MAR), intraocular pressure measurement by an air puff tonometer (Tonoref III, Nidec Co. Ltd, Aichi, Japan), slit lamp biomicroscopy, axial length measurement (AL-SCAN, Nidek Co. Ltd, Aichi, Japan), macular and peripapillary thickness measurement by SD-OCT, and FAZ and VD quantification using OCT-A (RS-3000 Advance, Nidek Co. Ltd, Gamagori, Japan). All participants also underwent an assessment of VEPs. The SD-OCT, OCT-A, and VEPs parameters were recorded.

After obtaining all the data, the subjects were divided into two groups: MS, and healthy controls. MS group was then divided into two subgroups: $\mathrm{MS}-\mathrm{ON}$ (eyes without an $\mathrm{ON}$ history) and MS + ON (eyes with an ON history).

\section{VEPs}

The International Society for Clinical Electrophysiology of Vision guidelines were used for VEP recordings [14]. With the subject sitting $1 \mathrm{~m}$ away from a monitor in a dark room, VEPs were recorded by eliciting the waveforms using a checkerboard pattern of 48 black and white squares reversed at $1 \mathrm{~Hz}$. The mean luminance of the checkerboard was $50 \mathrm{~cd} \mathrm{~m}^{-2}$, and the contrast between the black and white squares was $99 \%$. The reference electrode was placed on the midline frontal point $(\mathrm{Fz})$, an active electrode on the midline at the occipital area $(\mathrm{Oz}$ according to the 10-20 system), and the ground electrode on the forearm. Silver cup electrodes were used, and their impedance was maintained below $5 \mathrm{k} \Omega$. The responses were analysed using Medelec Synergy with a $1-100 \mathrm{~Hz}$ bandpass filter and a sweep time of $500 \mathrm{~ms}$. The stimuli were presented uni-ocularly. A hundred responses were averaged in every run, and two runs were performed for each eye. The latency and amplitude (peak to peak) of the P100 component were determined for each eye. The normative value was $100 \pm 10 \mathrm{~ms}$ for the VEP latency and $\geq 5 \mu \mathrm{V}$ for the VEP amplitude. Only the latency value was recorded for this study.

\section{Scan protocol}

We used Nidek's RS-3000 Advance system and updated AngioScan software (Navis ver. 1.8.0.) to collect and analyse SD-OCT and OCT-A images. This device operates at a central wavelength of $880 \mathrm{~nm}$ and a speed of A-scan rate of $53,000 \mathrm{scan} / \mathrm{s}$. The axial and transverse scan resolution in tissue is 7 and $20 \mu \mathrm{m}$, respectively. The scan range is $3-12$ $\mathrm{mm}$ horizontally, 3-9 mm vertically, and $2.1 \mathrm{~mm}$ in depth. In addition, the updated software allows removing the projection artefacts of the OCT-A scans by "ALL Layers" PAR feature.

Each eye was dilated with $1 \%$ tropicamide eye drop (Tropamid, Bilim Ilac Ltd, Istanbul, Turkey) and scanned by an experimented operator. The scanning was analysed in alignment with APOSTEL recommendations and normative data assessment study of the Nidek RS-3000 Advance device's new software $[15,16]$. We modified the OSCARIB quality control criteria for our device and checked the OCT scans before including them to the study $[17,18]$. The scanning was repeated if the SSI quality was $<7 / 10$. The scans included a $3 \times 3 \mathrm{~mm}$ macula map centred on the fovea and a $2.4 \times 4 \mathrm{~mm}$ disc map centred on the optic nerve head. The tracing HD plus function of the device allows reducing movement and blinking artefacts and continuing the examination after rest if the subject develops fatigue. After completing the scanning procedure, the following data were recorded: macular thickness (MT), GCL thickness (GCLT), 
RNFL thickness (RNFLT), FAZ metrics, and VD of the macula and peripapillary area. RNFLT was recorded at four different quadrants according to the TSNIT chart. The ETDRS chart in the macula map was used to obtain MT. GCLT was recorded for superior/inferior sectors of the macula. VD was defined as the percentage of the vascularized tissue within the surrounding area. The quantitative analysis of VD was performed with the coloured VD maps of the macula (ETDRS chart) and optic nerve head (TSNIT chart) (Fig. 1). Using the macular OCT-A scans, the FAZ area, perimeter, and circularity index (CI, values closer to " 1 " indicating a higher circularity) at the level of the superficial capillary plexus (SCP) (Supplementary Fig. 1) and the VD of SCP and deep capillary plexus (DCP) were recorded. The distribution of the VD was determined in accordance with the ETDRS 9 sectors chart. The retinal peripapillary capillary plexus (RPCP) VD for the TSNIT sectors were obtained from the OCT-A scans of the peripapillary area.

\section{Statistical analyses}

Quantitative variables were described as mean and standard deviation (SD), and qualitative variables as percentages. Power calculation was not calculated as the study was exploratory. The Shapiro-Wilk test was used to determine whether the sample came from a normally distributed population. The Pearson chi-square test was used to analyse the gender distribution among the groups. According to the normality test results, the Mann-Whitney $U$ test or Student's $t$ test was used to compare the independent samples. For the eye related parameters, each participant's average value of right and left eyes was used while comparing the MS and healthy control groups. The correlations between age, MS duration, relapse count, EDSS score, average value of right and left eyes' VEPs, MT, RNFLT, GCLT, FAZ metrics, and VD including SCP, DCP, and RPCP were analysed using the Pearson correlation coefficient. For the MS subgroups, generalized estimating equation (GEE) was used to check the associations of the ON history with the VEPs, structural OCT, and OCT-A parameters. A single GEE model with working correlation matrix "exchangeable" was created for each parameter as a dependent variable and the history of $\mathrm{ON}$ as the main effect (ordinal factor). Two final GEE models, corrected for age, gender, and ON history, were used for associations between EDSS (with ordinal logistic type of model) and BCVA (with linear type of model) with the duration of MS, VEPs latency, central foveal thickness (CFT), RNFLT, GCLT, global SCP, DCP and RPCP VDs and FAZ area to account for within-individuals inter-eye effects. The parameters with non-significant results were sequentially removed from these final models until only the significant ones remained.

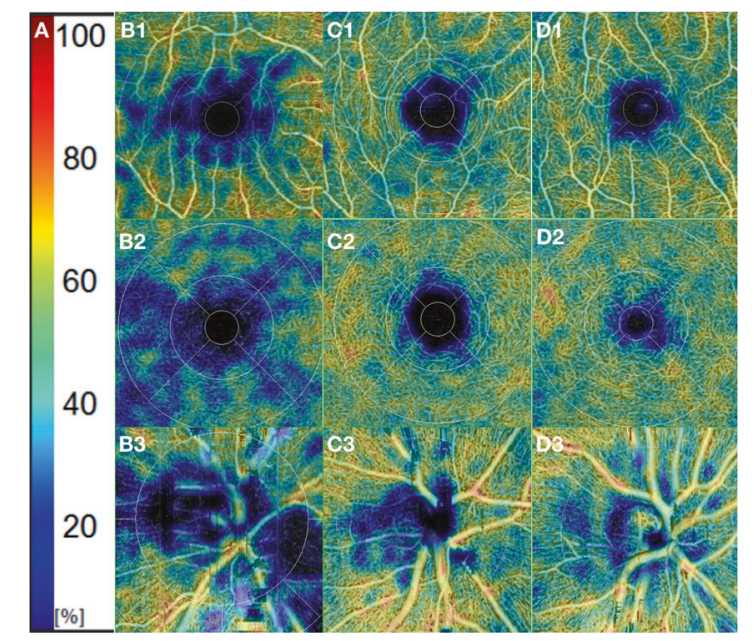

Fig. 1 Vessel density of $\operatorname{SCP}(B 1, \mathrm{C1}, \mathrm{D} 1), \operatorname{DCP}(\mathrm{B} 2, \mathrm{C} 2, \mathrm{D} 2)$, and $\mathbf{R P C P}(\mathbf{B 3}, \mathbf{C 3}, \mathbf{D 3})$. a Colour scale of the vessel density in percentages. b Vessel density of an MS patient with history of optic neuritis. c Vessel density of an MS patient without history of optic neuritis. d Vessel density of a healthy subject.

GEE results are given with regression coefficient $(B)$ and 95\% Wald confidence interval $[19,20]$.

All statistical analyses were performed using SPSS v. 21 (SPSS, Inc., Chicago, IL, USA). A $P$ value of less than 0.05 was considered statistically significant.

\section{Results}

\section{Study population and demographic data}

Fifty-one MS patients (102 eyes) and 61 healthy volunteers (122 eyes) were included in this study. Four MS patient's eight eyes were excluded from the study because they did not meet the OSCAR-IB quality control criteria (two eyes for SSI quality $<7$ and six eyes for inappropriate centralization of the optic nerve head. Thirty-five eyes of the MS patients had an ON history. The groups showed a similar distribution concerning gender and age. The demographic data are shown in Table 1 . There was no significant difference between the MS-ON and $\mathrm{MS}+\mathrm{ON}$ groups in terms of disease duration and EDSS score $(P=$ 0.685 and 0.817 respectively, GEE). The number of relapses within the last year was significantly greater in the $\mathrm{MS}+\mathrm{ON}$ group than in MS-ON $(P<0.001, t$-test $)$. The BCVA was significantly lower in the MS group than in healthy controls $(P=0.012$, GEE). However, it did not significantly differ between the MS-ON and $\mathrm{MS}+\mathrm{ON}$ subgroups $(P=0.150$, GEE).

In this study we gathered all the information of the therapies that the patients with MS get. Eight different immunotherapy regimens were recorded: interferon beta $1 \mathrm{~b}$ $0.3 \mathrm{mg}(n=5)$, interferon beta $1 \mathrm{a} 30 \mathrm{mcg}(n=7)$ and 44 
Table 1 Demographic and clinical data of the study groups.

\begin{tabular}{llll}
\hline & $\begin{array}{l}\text { MS } \\
n=47\end{array}$ & $\begin{array}{l}\text { HC } \\
n=61\end{array}$ & $P$ \\
\hline Female (n) & 39 & 49 & 0.68 \\
Age (years; mean \pm SD) & $38.29 \pm 8.71$ & $38.62 \pm 11.51$ & 0.36 \\
$\begin{array}{l}\text { Disease duration (years; } \\
\text { mean } \pm \text { SD) }\end{array}$ & $8.14 \pm 4.19$ & $\mathrm{n} / \mathrm{a}$ & \\
EDSS (median/range) & $1.50 / 0-5.50$ & $\mathrm{n} / \mathrm{a}$ & \\
No. of relapse within last & $0.54 \pm 0.79 / 0 /$ & $\mathrm{n} / \mathrm{a}$ & \\
year (mean/median/range) & $0-3$ & & \\
ON history (eyes) & 35 & $\mathrm{n} / \mathrm{a}$ & \\
VEP latency & $125.07 \pm 14.84$ & $106.58 \pm 0.88$ & $<0.001^{*}$ \\
(ms; mean \pm SD) & & & $0.08^{* *}$ \\
BCVA (logMAR; mean \pm & $0.02 \pm 0.007 /$ & $0.00 \pm 0.00 /$ & \\
SD/range) & $0.00-0.4$ & $0.00-0.00$ & \\
\hline
\end{tabular}

$S D$ standard deviation, EDSS expanded disability status scale, VEP visual evoked potential, $B C V A$ best-corrected visual acuity, $M S$ multiple sclerosis, $H C$ healthy controls, $O N$ optic neuritis, $n / a$ not applicable

*Mann-Whitney $U$ test

**Generalised estimating equation

mcg $(n=10)$, teriflunomide $14 \mathrm{mcg}(n=4)$, fingolimod $0.56 \mathrm{mg}(n=6)$, glatimer asetat $20 \mathrm{mg}(n=5)$, dimetil fumarate $240 \mathrm{mg}(n=6)$, and natalizumab $300 \mathrm{mg}(n=4)$.

The RNFLT for all the TSNIT sectors, MT accordance to the ETDRS chart, and GCLT for the superior and inferior hemifields significantly differed between the MS and healthy control groups $(P<0.05$, $t$-test, Table 2$)$. The CFT was significantly lower in the MS group than healthy control group $(P<0.001, t$-test) but the difference between the MS-ON and $\mathrm{MS}+\mathrm{ON}$ subgroups was weak, the CFT was tended to be lower in the $\mathrm{MS}+\mathrm{ON}$ subgroup. $(P=0.079, \mathrm{GEE})$.

\section{The OCT-A scan analyses; FAZ metrics}

There were no significant differences between the groups in terms of the FAZ area, perimeter, and CI $(P=0.762,0.673$, 0.710 respectively, Mann-Whitney $U$ test, Table 3$)$. All FAZ metrics were inversely correlated with CFT $\left(R^{2}=-0.309, P\right.$ $<0.001$ for the FAZ area, $R^{2}=-0.053, P=0.003$ for the perimeter, and $R^{2}=-0.025, P=0.040$ for the CI, Pearson's correlation test). The FAZ area and perimeter were inversely correlated with the VD of both SCP and DCP inner rings $(P$ $<0.05$, Pearson's correlation test), but not with the outer rings ( $P>0.05$, Pearson's correlation test). The FAZ area was not found to be associated with EDSS score or BCVA $(P=0.909,0.152$, respectively, GEE, Table 4).

\section{The OCT-A scan analyses; macular VD}

For all the ETDRS sectors of the macula, the VDs of SCP and DCP significantly differed between the study groups
$(P<0.05, t$-test, Table 3$)$. The VD of the global SCP was positively correlated with RNFLT in all sectors, MT in the ETDRS inner ring sectors, CFT, GCLT, and the VD of the temporal and inferior sectors of RPCP, and inversely correlated with age, and the FAZ area and perimeter $(P<0.05$, Pearson's correlation test). The VD of the global DCP was positively correlated with CFT and GCLT and inversely correlated with the FAZ area and perimeter $(P<0.05)$. The VD of DCP was also inversely correlated with VEP latency $\left(R^{2}=-0.068, P=0.001\right.$, Pearson's correlation test). No relation was found between EDSS score and BCVA and macular VD parameters $(P>0.05$, GEE, Table 4$)$.

\section{The OCT-A scan analyses; peripapillary VD}

The VD of the global RPCP was not significantly different among the study groups. Only the VD of the temporal quadrant significantly differed between the MS and healthy control groups $(P<0.001, t$-test $)$. The VD of the temporal sector was positively correlated with the RNFLT of all the TSNIT sectors, MT of all the ETDRS sectors except CMT, GCLT of the superior and inferior hemifields, and the VDs of SCP (except that of the inner ring) and DCP. A correlation was detected between the VD of the RPCP and BCVA $\left(R^{2}=-0.034, \quad P=0.041\right.$, Pearson's correlation test), however, it was disappeared after GEE analysis $(P=$ 0.891, Table 4).

\section{The VEPs analyses}

Significant differences were in the VEP latency between the MS and healthy control groups $(P<0.001$, Mann-Whitney $U$ test, Table 1). The mean latency was the shortest in healthy controls $(106.14 \pm 6.77)$ and longest in the MS + ON subgroup $(133.85 \pm 11.00)$. The VEP latency was inversely correlated with the RNFLT of all the TSNIT sectors, MT of all the ETDRS sectors, GCLT of the superior and inferior hemifields, the VD of the outer ring of SCP, and the VD of the DCP of all the ETDRS sectors $(P<$ 0.001 , Pearson's correlation test). The VEP latency did not have a significant relation with EDSS score, but worse vision found associated with longer VEP latency $(P=$ 0.008, GEE, Table 4).

\section{GEE results of the EDSS and BCVA}

The GEE results of the EDSS and BCVA models are presented in the Table 4. EDSS found associated with the duration of the MS, RNFLT, and CFT $(P<0.001,=0.011$, 0.045 respectively). The RNFLT and CFT were also associated with BCVA $(P=0.002,0.049$ respectively). In addition, the VEPs latency was found associated with BCVA $(P=0.008)$. 
Table 2 Structural optical coherence tomography data of the study groups.

\begin{tabular}{|c|c|c|c|c|c|}
\hline & $\begin{array}{l}\text { MS } \\
n=94 \text { eyes }\end{array}$ & $\begin{array}{l}\mathrm{HC} \\
n=122 \text { eyes }\end{array}$ & $\begin{array}{l}\mathrm{MS}-\mathrm{ON} \\
n=59 \text { eyes }\end{array}$ & $\begin{array}{l}\mathrm{MS}+\mathrm{ON} \\
n=35 \text { eyes }\end{array}$ & $P$ \\
\hline \multicolumn{6}{|l|}{ RNFLT } \\
\hline Global & $90.80 \pm 17.62$ & $106.88 \pm 11.14$ & $96.22 \pm 16.10$ & $81.68 \pm 16.46$ & $<0.001 * /<0.001 * *$ \\
\hline Superior & $113.62 \pm 17.62$ & $136.52 \pm 18.29$ & $119.88 \pm 20.21$ & $103.08 \pm 20.82$ & $<0.001 * /<0.001 * *$ \\
\hline Nasal & $69.46 \pm 17.62$ & $78.45 \pm 15.58$ & $72.25 \pm 15.83$ & $64.77 \pm 24.41$ & $0.001 * /<0.001 * *$ \\
\hline Inferior & $117.08 \pm 17.62$ & $139.77 \pm 19.53$ & $123.15 \pm 28.18$ & $106.85 \pm 17.64$ & $<0.001 * / 0.043^{* *}$ \\
\hline Temporal & $60.63 \pm 17.62$ & $72.68 \pm 12.82$ & $65.03 \pm 13.93$ & $53.22 \pm 17.64$ & $<0.001 * / 0.005^{* *}$ \\
\hline \multicolumn{6}{|l|}{ MT } \\
\hline Outer ring superior & $313.09 \pm 17.39$ & $332.15 \pm 15.24$ & $318.77 \pm 16.68$ & $303.51 \pm 14.21$ & $<0.001 * / 0.001 * *$ \\
\hline Outer ring nasal & $317.91 \pm 21.84$ & $341.27 \pm 15.57$ & $324.93 \pm 21.70$ & $306.08 \pm 16.50$ & $<0.001 * /<0.001 * *$ \\
\hline Outer ring inferior & $308.69 \pm 22.98$ & $328.70 \pm 18.27$ & $314.59 \pm 22.80$ & $298.74 \pm 19.87$ & $<0.001 * /<0.001 * *$ \\
\hline Outer ring temporal & $302.20 \pm 24.42$ & $323.10 \pm 16.35$ & $307.59 \pm 25.41$ & $293.11 \pm 19.85$ & $<0.001 * / 0.001 * *$ \\
\hline Inner ring superior & $324.60 \pm 21.98$ & $349.14 \pm 14.22$ & $331.44 \pm 22.15$ & $313.08 \pm 16.34$ & $<0.001 * / 0.005^{* *}$ \\
\hline Inner ring nasal & $319.28 \pm 20.55$ & $343.68 \pm 18.22$ & $327.18 \pm 18.64$ & $305.97 \pm 16.49$ & $<0.001 * /<0.001 * *$ \\
\hline Inner ring inferior & $319.64 \pm 25.76$ & $341.98 \pm 16.16$ & $326.86 \pm 25.93$ & $307.48 \pm 20.65$ & $<0.001 * /<0.001 * *$ \\
\hline Inner ring temporal & $309.76 \pm 19.46$ & $328.12 \pm 17.41$ & $315.61 \pm 18.04$ & $299.91 \pm 18.03$ & $<0.001 * /<0.001 * *$ \\
\hline CFT & $249.29 \pm 16.83$ & $264.45 \pm 22.88$ & $251.64 \pm 18.31$ & $245.38 \pm 13.19$ & $<0.001 * / 0.079 * *$ \\
\hline \multicolumn{6}{|l|}{ GCLT } \\
\hline Superior & $91.20 \pm 14.88$ & $109.63 \pm 8.04$ & $96.86 \pm 12.10$ & $81.65 \pm 14.38$ & $<0.001 * /<0.001 * *$ \\
\hline Inferior & $90.89 \pm 17.22$ & $109.43 \pm 9.24$ & $97.62 \pm 13.40$ & $79.54 \pm 17.14$ & $<0.001 * /<0.001 * *$ \\
\hline
\end{tabular}

RNFLT retinal nerve fibre layer thickness, $M T$ macular thickness, $C F T$ central foveal thickness, $G C L T$ ganglion cell layer thickness, $M S$ multiple sclerosis, $M S-O N$ MS without history of $\mathrm{ON}, M S+O N \mathrm{MS}$ with history of $\mathrm{ON}, H C$ healthy controls, $O N$ optic neuritis

*Comparison of MS with $\mathrm{HC}$ using $t$-test

**Comparison of MS $-\mathrm{ON}$ with MS + ON using generalised estimating equation

\section{Discussion}

Considering the growing interest in the contribution of vascular disorders to the MS pathogenesis, in this study, we presented the OCT-A findings of the MS cases. Several studies showed that the retinal VD of both macular and peripapillary areas were reduced in MS patients $[9,11,12,21]$. To our knowledge, this study is the first to give information about the FAZ metrics in MS subjects. Given the fact that the commercially available OCT-A devices use different algorithms and software with different features, all devices should have its own normative data and there is a need of standardization for numerous disorders [22]. This study is also the first study about the relation of MS with retinal microvascular structure that Nidek's RS3000 Advance device and it's new software is used.

A recent study showed that increased FAZ area and decreased circularity were associated with the severity of diabetic retinopathy. Decreased circularity was also found associated with the reduction of visual acuity [23]. The authors suggested that microvascular damage might be the reason for these changes. Since the main aim of OCT-A studies was to investigate the role of vascular involvement in the pathogenesis of MS, we evaluated the FAZ area along with the perimeter and circularity. We found that the FAZ metrics did not significantly differ between the MS subgroups and healthy controls (Table 3). The FAZ metrics' results of our study suggests the second hypotheses rather than the microvascular damage; reduced metabolic demand in atrophied central nervous system causes the hypoperfusion [24]. Further studies with repeated measurements over a longer follow-up could provide more detailed information about the FAZ metrics in MS.

Some researchers revealed hypoperfusion in the brain's grey and white matter $[25,26]$. Reduced VD in the macular and peripapillary areas could be the reflection of global brain hypoperfusion [27]. We found that the VD of the macula was reduced in MS patients, as also suggested by previous studies (Table 3) [9, 11, 21, 28]. The VD of the MS patients was significantly lower compared with the VD of the healthy control group. Similarly, the VD of the MS + ON subgroup was significantly lower compared with the VD of the MS-ON subgroup. Lanzillo et al. and Feucht et al. reported that the VD of the MS patients without ON was significantly reduced compared with healthy controls $[9,28]$. We did not investigate the difference between MS-ON/MS + ON subgroups with the healthy control group because of the rather small sample size. We only 
Table 3 Optical coherence tomography angiography data of the study groups.

\begin{tabular}{|c|c|c|c|c|c|}
\hline & $\begin{array}{l}\text { MS } \\
n=94 \text { eyes }\end{array}$ & $\begin{array}{l}\mathrm{HC} \\
n=122 \text { eyes }\end{array}$ & $\begin{array}{l}\mathrm{MS}-\mathrm{ON} \\
n=59 \text { eyes }\end{array}$ & $\begin{array}{l}\mathrm{MS}+\mathrm{ON} \\
n=35 \text { eyes }\end{array}$ & $P$ \\
\hline \multicolumn{6}{|c|}{ FAZ metrics } \\
\hline Area & $0.34 \pm 0.12$ & $0.30 \pm 0.09$ & $0.33 \pm 0.13$ & $0.34 \pm 0.11$ & $0.762 * / 0.621 * *$ \\
\hline Perimeter & $2.89 \pm 0.63$ & $2.89 \pm 0.58$ & $2.84 \pm 0.65$ & $2.99 \pm 0.59$ & $0.673 * / 0.274 * *$ \\
\hline $\mathrm{CI}$ & $0.49 \pm 0.85$ & $0.49 \pm 0.074$ & $0.50 \pm 0.008$ & $0.47 \pm 0.07$ & $0.710 * / 0.109 * *$ \\
\hline \multicolumn{6}{|l|}{ SCP VD } \\
\hline Global & $40.06 \pm 4.86$ & $42.35 \pm 2.68$ & $41.25 \pm 4.42$ & $38.05 \pm 4.97$ & $<0.001 * / 0.002 * *$ \\
\hline Inner ring & $37.23 \pm 6.64$ & $39.81 \pm 4.58$ & $38.49 \pm 6.51$ & $35.11 \pm 6.38$ & $0.006^{*} / 0.016^{* *}$ \\
\hline Outer ring & $52.01 \pm 4.43$ & $54.08 \pm 1.56$ & $53.15 \pm 2.79$ & $50.08 \pm 5.87$ & $<0.001 * / 0.001 * *$ \\
\hline \multicolumn{6}{|l|}{ DCP VD } \\
\hline Global & $33.73 \pm 6.79$ & $38.21 \pm 4.53$ & $34.69 \pm 5.96$ & $32.11 \pm 7.81$ & $<0.001 * / 0.075^{* *}$ \\
\hline Inner ring & $27.65 \pm 8.11$ & $32.92 \pm 7.69$ & $28.61 \pm 7.87$ & $32.11 \pm 7.81$ & $<0.001 * / 0.141 * *$ \\
\hline Outer ring & $48.08 \pm 7.98$ & $52.10 \pm 3.17$ & $49.06 \pm 6.48$ & $46.42 \pm 9.91$ & $<0.001 * / 0.122^{* *}$ \\
\hline \multicolumn{6}{|l|}{ RPCP VD } \\
\hline Global & $53.59 \pm 3.69$ & $54.24 \pm 2.23$ & $53.47 \pm 3.66$ & $53.80 \pm 3.79$ & $0.072 * / 0.682 * *$ \\
\hline Superior & $54.54 \pm 2.85$ & $55.50 \pm 2.55$ & $54.32 \pm 5.57$ & $56.38 \pm 4.30$ & $0.271 * / 0.414 * *$ \\
\hline Inferior & $54.22 \pm 2.97$ & $54.41 \pm 3.17$ & $49.72 \pm 5.44$ & $50.54 \pm 4.90$ & $0.697 * / 0.485^{* *}$ \\
\hline
\end{tabular}

$F A Z$ foveal avascular zone, $C I$ circularity index, $V D$ vessel density, $S C P$ superficial capillary plexus, $D C P$ deep capillary plexus, $R P C P$ retinal peripapillary capillary plexus, $M S$ multiple sclerosis, $M S-O N$ MS without history of ON, $M S+O N$ MS with history of ON, $H C$ healthy controls, $O N$ optic neuritis *Comparison of MS with $\mathrm{HC}$ using $t$-test

**Comparison of MS-ON with MS + ON using generalised estimating equation

Table 4 The GEE models for EDSS and BCVA.

\begin{tabular}{|c|c|c|c|}
\hline & $B$ & $\begin{array}{l}95 \% \text { Wald } \\
\text { confidence interval }\end{array}$ & $P$ \\
\hline \multicolumn{4}{|l|}{ EDSS } \\
\hline Duration of MS & 0.142 & $0.092 / 0.192$ & $<0.001$ \\
\hline VEP latency & 0.000 & $-0.026 / 0.026$ & 0.989 \\
\hline The global RNFLT & -0.002 & $-0.035 /-0.005$ & 0.011 \\
\hline CFT & -0.024 & $-0.041 /-0.008$ & 0.045 \\
\hline FAZ area & 0.078 & $-3.23 / 2.88$ & 0.909 \\
\hline SCP global VD & 0.008 & $-0.031 / 0.047$ & 0.692 \\
\hline DCP global VD & 0.007 & $-0.033 / 0.047$ & 0.736 \\
\hline RPCP global VD & -0.008 & $-0.041 /-0.008$ & 0.733 \\
\hline \multicolumn{4}{|l|}{ BCVA } \\
\hline Duration of MS & -0.002 & $-0.066 / 0.003$ & 0.443 \\
\hline VEP latency & 0.002 & $-0.003 / 0.002$ & 0.008 \\
\hline The global RNFLT & -0.002 & $-0.004 /-0.001$ & 0.002 \\
\hline CFT & -0.001 & $-0.003 / 0.000$ & 0.049 \\
\hline FAZ area & -0.119 & $-0.281 / 0.044$ & 0.152 \\
\hline SCP global VD & -0.002 & $-0.006 / 0.003$ & 0.443 \\
\hline DCP global VD & -0.001 & $-0.004 / 0.001$ & 0.297 \\
\hline RPCP global VD & 0.000 & $-0.004 / 0.005$ & 0.891 \\
\hline
\end{tabular}

$G E E$ generalized estimating equation, $E D S S$ expanded disability status scale, $B C V A$ best-corrected visual acuity, $M S$ multiple sclerosis, RNFLT retinal nerve fibre layer thickness, CFT central foveal thickness, $F A Z$ foveal avascular zone, $V D$ vessel density, $S C P$ superficial capillary plexus, $R P C P$ retinal peripapillary capillary plexus examined patients with the relapsing-remitting subtype of MS in our study while previous studies included different subtypes of MS.

With the technological developments and updated software, the segmentation of the peripapillary region has become possible. In the present study, only the VD of the RPCP temporal sector was significantly reduced among the study groups. Similarly, Kumar et al. and Holló showed a significant reduction in the VD of only superotemporal and inferotemporal sectors, associated with visual field loss in glaucoma patients [29, 30]. A possible reason for the nonsignificant changes obtained from the other sectors is that the vascular structure of optic nerve head and peripapillary region mainly consists of large vessels and might have masked microvascular damage on the OCT-A scan [31, 32]. In another study reporting the 2-year follow-up results of the peripapillary VD in patients with glaucoma, Holló found that the peripapillary VD did not significantly differ compared with the baseline values in patients with thinned RNFLT due to the progression of the disease. However, after the removal of large vessels by software, the authors noted a significant reduction in the peripapillary VD [33]. Further studies using software that removes large vessels are needed to provide an understanding of the importance of the VD of RPCP in MS cases.

Di Maggio et al. published the results of a comparative study of VEPs and RNFLT in MS subjects and showed that 
the RNFLT and VEP score were inversely correlated. They suggested that RNFLT and VEPs had similar sensitivity according to the abnormalities in eyes with an ON history [2]. We similarly found that the VEP latency was inversely correlated with MT (including CFT), GCLT, and the VDs of the SCP outer ring and DCP in all sectors. To our knowledge, our study is the first that investigated the relations between VEPs and VD among MS patients.

EDSS score and BCVA are important parameters for the prognosis of MS. Lanzillo et al. reported that EDSS was correlated with VD, and Leocani et al. and Di Maggio et al. suggested the same for VEPs [2, 9, 34]. In our final GEE model, we only found associations between EDSS score with MS duration, RNFLT, and CFT. The main limitation of this study in terms of EDSS could be the distribution of the EDSS scores. Only 26 of 94 eyes had an EDSS score greater than 2. In future, studies including populations with normally distributed EDSS scores could provide more precise associations with other parameters. The distribution was also a limitation for BCVA, with only 11 eyes having a BCVA lower than 20/20. Thus, the BCVA was found to be associated with only RNFLT, CFT, and VEP latency.

The present study has several limitations. First limitation is the rather small sample size. Secondly, we could not have analysed the effect of the immunotherapy that the patients get. Further studies with larger sample sizes are needed to analyse the effects of the treatment strategies on retinal microvascular network. Another limitation is since this is an exploratory study, no adjustment for multiple comparisons was made, therefore it is impossible for us to draw definite conclusions.

In conclusion, in this paper, we presented the OCT-A findings of MS patients along with the structural OCT and VEP parameters. However, considering that it is almost impossible to determine the exact role of vascular involvement in the pathogenesis of MS using cross-sectional studies, we are currently performing a follow-up study to better explain our findings.

\section{Summary}

\section{What was known before}

- Structural OCT findings are correlated with the MS disability score.

\section{What this study adds}

- Optic coherence tomography angiography findings in subjects with MS is correlated with the structural OCT findings. Additional follow-up studies needed.

\section{Compliance with ethical standards}

Conflict of interest The authors declare that they have no conflict of interest.

Publisher's note Springer Nature remains neutral with regard to jurisdictional claims in published maps and institutional affiliations.

\section{References}

1. Britze J, Frederiksen JL. Optical coherence tomography in multiple sclerosis. Eye. 2018;32:884-8.

2. DI Maggio G, Santangelo R, Guerrieri S, Bianco M, Ferrari L, Medaglini S, et al. Optical coherence tomography and visual evoked potentials: which is more sensitive in multiple sclerosis? Mult Scler. 2014;20:1342-7.

3. Kerrison JB, Flynn T, Green WR. Retinal pathologic changes in multiple sclerosis. Retina. 1994;14:445-51.

4. Petzold A, Balcer L, Calabresi PA, Costello F, Frohman T, Frohman E, et al. Retinal layer segmentation in multiple sclerosis: a systematic review and meta-analysis. Lancet Neurol. 2017; 16:797-812.

5. Oertel FC, Zimmermann HG, Brandt AU, Paul F. Novel uses of retinal imaging with optical coherence tomography in multiple sclerosis. Expert Rev Neurother. 2019;19:31-43.

6. Galetta SL, Villoslada P, Levin N, Shindler K, Ishikawa H, Parr E, et al. Acute optic neuritis: unmet clinical needs and model for new therapies. Neurol Neuroimmunol NeuroInflammation. 2015;2:1-11.

7. Oberwahrenbrock T, Traber GL, Lukas S, Gabilondo I, Nolan R, Songster C, et al. Multicenter reliability of semiautomatic retinal layer segmentation using OCT. Neurol Neuroimmunol Neuroinflammation. 2018;5:1-7.

8. Petzold A, Wattjes MP, Costello F, Flores-Rivera J, Fraser CL, Fujihara $\mathrm{K}$, et al. The investigation of acute optic neuritis: a review and proposed protocol. Nat Rev Neurol. 2014;10:447-58.

9. Lanzillo R, Cennamo G, Criscuolo C, Carotenuto A, Velotti N, Sparnelli $\mathrm{F}$, et al. Optical coherence tomography angiography retinal vascular network assessment in multiple sclerosis. Mult Scler J. 2018;24:1706-14.

10. Wang L, Murphy O, Caldito NG, Calabresi PA, Saidha S. Emerging applications of optical coherence tomography angiography (OCTA) in neurological research. Eye Vis. 2018;5:1-11.

11. Spain RI, Liu L, Zhang X, Jia Y, Tan O, Bourdette D, et al. Optical coherence tomography angiography enhances the detection of optic nerve damage in multiple sclerosis. Br J Ophthalmol. 2018;102:520-4.

12. Wang X, Jia Y, Spain R, Potsaid B, Liu JJ, Baumann B, et al. Optical coherence tomography angiography of optic nerve head and parafovea in multiple sclerosis. Br J Ophthalmol. 2014; 98:1368-73.

13. Polman $\mathrm{CH}$, Reingold SC, Banwell B, Clanet M, Cohen JA, Filippi M, et al. Diagnostic criteria for multiple sclerosis: 2010 Revisions to the McDonald criteria. Ann Neurol. 2011; 69:292-302.

14. Odom JV, Bach M, Brigell M, Holder GE, McCulloch DL, Tormene AP, et al. ISCEV standard for clinical visual evoked potentials (2009 update). Doc Ophthalmol. 2010;120:111-9.

15. Cruz-Herranz A, Balk LJ, Oberwahrenbrock T, Saidha S, Martinez-Lapiscina EH, Lagreze WA, et al. The APOSTEL recommendations for reporting quantitative optical coherence tomography studies. Neurology. 2016;86:1-7.

16. Yilmaz H, Karakurt Y, Icel E, Ugurlu A, Ucak T, Tasli NG, et al. Normative data assessment of vessel density and foveal avascular 
zone metrics using AngioScan software. Curr Eye Res. 2019;0:1-8.

17. Tewarie P, Balk L, Costello F, Green A, Martin R, Schippling S, et al. The OSCAR-IB consensus criteria for retinal OCT quality assessment. PLoS ONE. 2012;7:1-7.

18. Schippling S, Balk LJ, Costello F, Albrecht P, Balcer L, Calabresi PA, et al. Quality control for retinal OCT in multiple sclerosis: validation of the OSCAR-IB criteria. Mult Scler J. 2015; 21:163-70.

19. Ayadi N, Dörr J, Motamedi S, Gawlik K, Bellmann-Strobl J, Mikolajczak J, et al. Temporal visual resolution and disease severity in MS. Neurol Neuroimmunol NeuroInflammation. 2018;5:1-8

20. Ying Gshuang, Maguire MG, Glynn R, Rosner B. Tutorial on biostatistics: statistical analysis for correlated binary eye data. Ophthalmic Epidemiol. 2018;25:1-12.

21. Lanzillo R, Cennamo G, Moccia M, Criscuolo C, Carotenuto A, Frattaruolo N, et al. Retinal vascular density in multiple sclerosis: a 1-year follow-up. Eur J Neurol. 2019;26:198-201.

22. Corvi F, Pellegrini M, Erba S, Cozzi M, Staurenghi G, Giani A. Reproducibility of vessel density, fractal dimension, and foveal avascular zone using 7 different optical coherence tomography angiography devices. Am J Ophthalmol. 2018;186:25-31.

23. Tang FY, Ng DS, Lam A, Luk F, Wong R, Chan C, et al. Determinants of quantitative optical coherence tomography angiography metrics in patients with diabetes. Sci Rep. 2017; $7: 1-10$.

24. Allen NB, Lichtman JH, Cohen HW, Fang J, Brass LM, Alderman MH. Vascular disease among hospitalized multiple sclerosis patients. Neuroepidemiology. 2008;30:234-8.

25. Doche E, Lecocq A, Maarouf A, Duhamel G, Soulier E, ConfortGouny $\mathrm{S}$, et al. Hypoperfusion of the thalamus is associated with disability in relapsing remitting multiple sclerosis. J Neuroradiol. 2017;44:158-64

26. Narayana PA, Zhou Y, Hasan KM, Datta S, Sun X, Wolinsky JS. Hypoperfusion and T1-hypointense lesions in white matter in multiple sclerosis. Mult Scler J. 2014;20:363-73.

27. D'Haeseleer M, Hostenbach S, Peeters I, El Sankari S, Nagels G, De Keyser J, et al. Cerebral hypoperfusion: a new pathophysiologic concept in multiple sclerosis? J Cereb Blood Flow Metab. 2015;35:1406-10.

28. Feucht N, Maier M, Lepennetier G, Pettenkofer M, Wetzlmair C, Daltrozzo $\mathrm{T}$, et al. Optical coherence tomography angiography indicates associations of the retinal vascular network and disease activity in multiple sclerosis. Mult Scler J. 2018;20:224-34.

29. Kumar RS, Anegondi N, Chandapura RS, Sudhakaran S, Kadambi SV, Rao HL, et al. Discriminant function of optical coherence tomography angiography to determine disease severity in glaucoma. Investig Ophthalmol Vis Sci. 2016;57:6079-88.

30. Holló G. Relationship between optical coherence tomography sector peripapillary angioflowdensity and Octopus visual field cluster mean defect values. PLoS ONE. 2017;12:1-12.

31. Bojikian KD, Chen PP, Wen JC. Optical coherence tomography angiography in glaucoma. Curr Opin Ophthalmol. 2019;30:110-6.

32. Moghimi S, Hou H, Rao H, Weinreb RN. Optical coherence tomography angiography and glaucoma: a brief review. Asia-Pac J Ophthalmol. 2019;8:115-25.

33. Holló G. Influence of removing the large retinal vessels-related effect on peripapillary vessel density progression analysis in glaucoma. J Glaucoma. 2018;27:e137-9.

34. Leocani L, Rovaris M, Boneschi FM, Medaglini S, Rossi P, Martinelli V, et al. Multimodal evoked potentials to assess the evolution of multiple sclerosis: a longitudinal study. J Neurol Neurosurg Psychiatry. 2006;77:1030-5. 